\section{Kerstin Schmit}

n der langen Geschichte der TCM existieren zahlreiche Aufzeichnungen und Beschreibungen von Tumorerkrankungen. Der Begriff „, liu“, als Inskription auf Knochen und Muscheln, kann bis in die Shang-Dynastie (16001100 v. Chr.) zurückverfolgt werden.

Später in Zhou Li (Zhou Rituals), aus dem 11. Jahrhundert v. Chr., werden Ärzte beschrieben, die sich auf die Behandlung von Tumoren spezialisiert hatten und als „Yang Doktor“ bezeichnet wurden. Noch heute werden in Korea und Japan Tumore Zhong Yang benannt.

$R u$ Yan war im alten China die Bezeichnung für Brustkrebs. Das Zeichen $r u$ steht für Brust, yan zeigt einen Felsen, übersetzt heisst ru yan „BrustStein“. Demzufolge handelt es sich um einen Knoten, der hart ist wie ein Stein oder Schildkrötenpanzer.

Folgendes Zitat eines Arztes aus der Ming-Dynastie macht deutlich, dass es sich dabei um Brustkrebs handelt: „Am Anfang ist er wie eine Bohne, dann wie ein Ei, ohne Schmerzen oder Jucken, nach einem Jahr kommen Schmerzen hinzu. Dann wächst er zu einem grossen Knoten an, die Haut wird purpurfarben, es bilden sich Geschwüre aus, der Knoten ist wie ein Stein, Blut tritt aus, die fünf Yin-Organe erschöpfen sich" [1].

\section{Pathomechanismen}

Gemäss der TCM ist Brustkrebs das Resultat eines Kontinuums von Erkrankungen, zu denen auch Spannung der Brust vor der Menstruation oder fibrozystische Brusterkrankungen zählen. Aus diesem Grund wird dem PMS in der TCM viel mehr Krankheitswert beigemessen als in der westlichen Medizin.

FLAWS und WOLFE halten emotionale

\title{
Das Mamma-Karzinom aus der Sicht der TCM
}

Etwa 50\% der Patientinnen mit Brustkrebs entscheiden sich dafür, die schulmedizinische Behandlung durch eine Form der komplementärmedizinischen Therapie zu ergänzen. Die Kombination von Schulmedizin mit Chinesischer Medizin bietet eine optimale Möglichkeit der ganzheitlichen Behandlung - nicht nur für Brustkrebspatientinnen. Nicht nur Diagnostik und Therapiemethoden unterscheiden sich, sondern der gesamte Therapieansatz. Im Unterschied zur Schulmedizin geht man von der aktuellen physischen und psychischen Konstitution der Patientin aus und das Resultat ist ein individuelles Behandlungskonzept. Dieser Beitrag soll einen kurzen Einblick geben, welche Pathomechanismen und Syndrome in der Chinesischen Medizin beim Mammakarzinom eine Rolle spielen und wie sie sich darstellen.

Störungen für einen der Grundstein einer neoplastischen Entwicklung [2]. Psychoemotionaler Stress, Frustration und Depression schwächen die Leber, die ihre Kontrollfähigkeit verliert und das Qi gerät ins Stocken. Die Folge sind erstens Stagnation und Akkumulation, zweitens verursacht die Qi-Stagnation Wärme und drittens der Gegenfluss das gegenläufige $Q i$. Das stagnierte $Q i$ greift auf horizontaler Ebene Milz und Magen an bzw. verstärkt eine schon bestehende Milz-Schwäche.

In der chinesischen Literatur wird schon lange eine Verbindung zwischen Mammakarzinom und einer Milz-Leber-Pathologie postuliert. Der durch Depression oder Ärger angespannte Leber-Qi-Fluss attackiert die Milz, die ihrer Transport- und Transformationsfunktion nicht mehr nachkommen kann. Auch wenn Wünsche über lange Zeit nicht erfüllt werden können, ist der Energiefluss in den Leitbahnen nicht mehr glatt und das Resultat sind Blockaden.

Stockt das Blut, kommt es zur BlutStase. Stagnieren die Körpersäfte, weil sie nicht mehr transformiert werden, bildet sich Schleim. Schleim führt zur Verlegung der Meridiane und Gefässe und dadurch wieder zu Stagnation und Retention von $Q i$ und Blut. Gestörter Qi-Fluss wiederum schädigt die Organe und kann so $\mathrm{zu}$ weiterer Bildung von
Schleim führen. Sowohl Schleim als auch Blut-Stase behindern den freien Fluss des Yang, das sich sammelt und zu Feuer werden kann.

Zwei alte Zitate beschreiben das folgendermassen: Yi Zong Jin Jian (The Golden Mirror of Medicine): "Hard nodes in the breast are due to retention, binding and stagnation of Qi in the Liver and Spleen channels. In mild cases they take the form of mammary lotus (ru lian), in severe cases, mammary rock (ru yan)" [3].

Auch in Fu Ren Da Quan Liang Fang (Complete Effektive Prescription of Women`s Diseases) steht: „Knoten in der Brust sind bedingt durch Depression und Wut im Funktionskreis Leber und Milz oder durch Mangel bzw. geschädigtes Qi und Blut" [3].

Das Qi des Chong Mai kann durch eine Leber-Qi-Blockade im Fluss behindert werden. Wenn auch Milz und Niere geschwächt sind, kommt es zu einer Funktionsstörung der Chong Mai. Er verteilt sich über die Brust ebenso wie die Leber- und Magen-Leitbahn, wobei der Leber-Meridian speziell die Mamille versorgt. In allen drei Leitbahnen können in diesem Bereich Blockaden entstehen.

Zur Pathogenese existieren natürlich diverse Theorien. DR. Sun BING-YAN, ein heute praktizierender Krebsspezialist, ist überzeugt, dass Toxine die 
ausschlaggebenden Faktoren darstellen. Sie entstehen aus einer Kombination von Hitze, Nässe und Blut-Stase, deren Entwicklung umso schneller verläuft, je stärker die Qi-Stagnation ist [4]. Seiner Ansicht nach sind vor allem Patienten mit kalter Konstitution betroffen, denen es an Yang in der Niere und Galle mangelt. Ist das Nieren-Yang zu schwach, kommen alle Qi-Funktionen im Körper ins Stocken und es wird für den Organismus schwierig, eine Krankheit zu bekämpfen.

Laut Prof. Hu von der Universitätsklinik für TCM in Chengdu ist die Wurzel einer Neoplasie eine Schwäche des aufrechten Zheng Qi [5]. Die Schutzfunktionen der unterschiedlichen $Q i$ Arten setzt sie dem Begriff der Immunabwehr des Körpers gleich. Aus der Schwäche des Zheng Qi resultieren folgende drei Zustände: Schleim, BlutStase und schlechte Verteilung der nährenden Substanzen. Wie ausgeprägt die Schwäche sein muss, ist unklar. Wenn das Zheng Qi noch stark genug ist, kann es sein, dass der Tumor benigne bleibt.

\section{Syndromdifferenzierung}

Heute wird entsprechend der nachfolgenden Pathologien differenziert $[3,6]$.

\section{Leber-Qi-Stagnation}

Eines der häufigsten Muster in der TCM überhaupt. Hauptsymtome sind feste Knoten, die Spannung und Schmerz verursachen, Gereiztheit, Appetitverlust, bitterer Mundgeschmack, trockener Rachen sowie prämenstruelles Syndrom. Die Zunge ist oft unverändert, der Zungenbelag kann gelblich, dünn, der Puls gespannt/drahtig, eventuell fadenförmig und dünn sein. Brustspannen vor der Menstruation, kombiniert mit Stimmungsschwankungen sowie Schmerzen in Brust und Mamille sind typisch, sodass sogar die Kleidung als unangenehm empfunden wird. In der westlichen Medizin handelt es sich dabei um PMS, eventuell auch um erste Zysten. Der Menstruationszyklus ist unregelmässig, die Blutung schmerzhaft, unter Umständen mit Koagel, begleitet von Reizbarkeit.

\section{Nässe/Schleim aufgrund eines Milz-Qi/Yang-Mangels}

Symptomatisch sind derbe, unregelmässige Schwellungen in Brust und Axilla, verbunden mit Ikterus, psychische und physische Erschöpfung, kalte Hände und Füsse, Oppressionsgefühl thorakal und epigastrisch, Schweregefühl, sowie breiige Stühle. Die Zunge erscheint blass mit Zahnimpressionen und weisslichem oder dickem weissen Belag, der Puls ist schlüpfrig und tief. Wenn die Schleim-Symptomatik überwiegt, kann sich der Knoten auch weich und gross, eventuell eiförmig darstellen. Aus westlicher Sicht sind oft Zysten oder Fibroadenome gemeint. $\mathrm{Zu}$ den allgemeinen Manifestationen gehören noch Schweregefühl, Appetitlosigkeit, Völlegefühl, Ödeme und blasse, fahle Gesichtsfarbe.

\section{Disharmonie von Chong und Ren Mai}

Chong Mai und Ren Mai sind 2 der 8 ausserordentlichen Meridiane. Ihr Ursprung liegt in den Nieren und sie gelten als Reservoir und sind für die Regulation einiger körperlicher Zyklen und Strukturen zuständig.

Manifestation in Form von derben, druckschmerzhaften Knoten, Menstruationsstörungen, wobei der Zyklus eher lang ist, begleitet von Dysmenorrhoe. Brustspannen prä- und postmenstruell und Schwellung bilden sich langsam zurück. Auch Schmerzen und Schwäche der LWS sowie der unteren Extremitäten, Kälte im Unterbauch, eventuell Infertilität, 5 heisse Herzen (Hitzegefühl an Handflächen, Fusssohlen, Brust), trockene Augen und Mund können hinzukommen. Der Zungenkörper ist blass mit dünnem weisslichen Belag, der Puls gespannt, eventuell auch schlüpfrig. Dieses Muster tritt häufiger bei Nullipara, nach mehreren Aborten auf oder wenn nicht gestillt wurde.

\section{Blut-Stase und Hitze-Toxine}

Es zeigen sich feste unbewegliche Verhärtungen, begleitet von brennenden Hitzesensationen und Schmerz. Die Haut darüber erscheint prall, dunkelrot und glanzlos, auch Ulzeration mit Sekretion kann auftreten. Ausserdem beklagen die Patienten subfebrile Temperaturen, Kurzatmigkeit, Gereiztheit,
Spasmen oder aufgeblähes Abdomen. In anderen Fällen ist eine anhaltend schmerzhaft fixierte Schwellung vordergründig, begleitet von bitterem Mundgeschmack, Völle- und Oppressionsgefühl im Oberbauch. Wiederkehrende intermittierende Blutungen oder anomale Mikrozirkulation ist möglich.

Die toxische Hitze speziell äussert sich in einer lokalen Schwellung mit starken Schmerzen. In der Schulmedizin entspricht dies eher einer Mastitis mit Abszess, kann aber auch auf ein Karzinom hinweisen. Es kann zum Austritt von Mamillarsekret kommen, das gelb oder blutig und übel riechend ist.

Da sich die Blut-Stase in Folge als sehr wichtiger Faktor herausstellte, soll darauf etwas näher eingegangen werden. Laut MACIOCIA ist das charakteristische Symptom der fixierte, stechende, bohrende Schmerz, der abends oder in der Nacht stärker wird [6]. Lippen und Gesicht können zyanotisch verfärbt sein, Menstruation dunkel und klumpig. An der Haut sind Varizen oder erweiterte Äderchen zu sehen. Die Zunge ist purpurfarben, zum Teil mit Staseflecken. Je nach Ausmass der Blut-Stase sind die Venen an der Zungenunterseite gestaut, prall gefüllt und verzweigt. Der Puls ist saitenförmig und rau. Wenn Blut-Mangel die Grundlage für die Stase ist, kann er auch fein und schwach sein.

\section{Mangel an Blut und $Q i$}

Das Muster im Tumorspätstadium ist charakterisiert durch Knoten, die anfangs als Erosion und später in Form von Ulzeration mit fauligem, dünnen, klaren Exsudat auffallen. Die Haut erscheint trocken, glanzlos, begleitet von mentaler Erschöpfung, Kurzatmigkeit, Kachexie, Blässe, Appetitlosigkeit, profusem Schwitzen und Schlafstörungen. Der Zungenkörper tritt blass in Erscheinung, der Belag gelb oder weiss und klebrig, der Puls kraftlos und tief.

\section{Yin-Mangel}

Der Knoten besteht meist schon seit längerer Zeit. Mamillarsekret von eitriger oder blutiger Qualität ist möglich. Subfebrile Temperatur nachmittags, Kachexie, heisse Handflächen und Fusssohlen, Obstipation, Schwindel, Trockenheit von Mund und Rachen, 


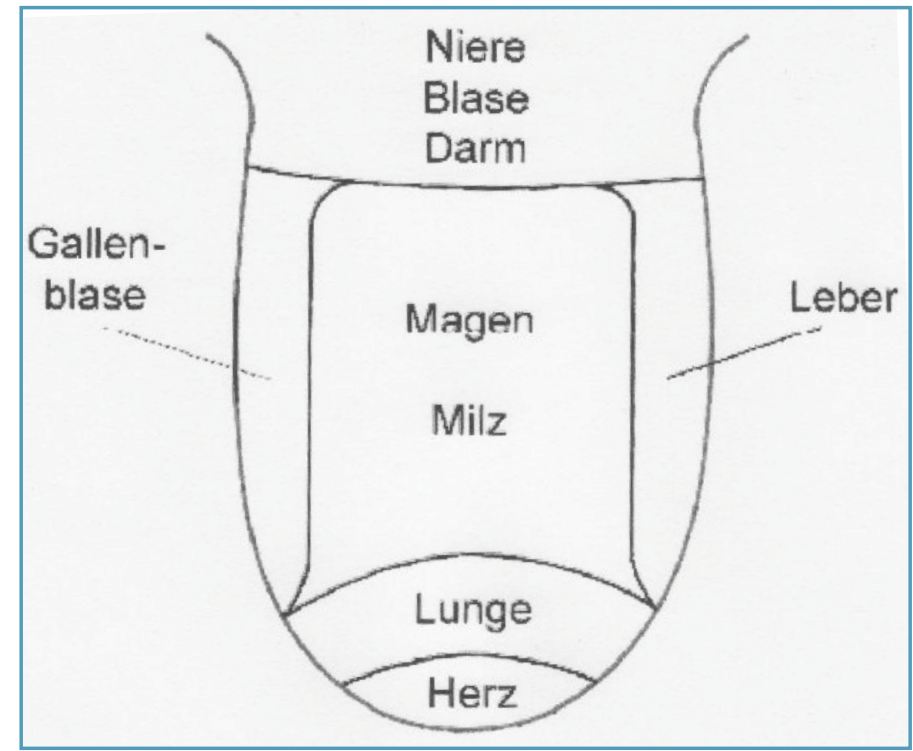

Abb. 1. Topographie der Zunge mit zugeordneten Organen, nach [6].

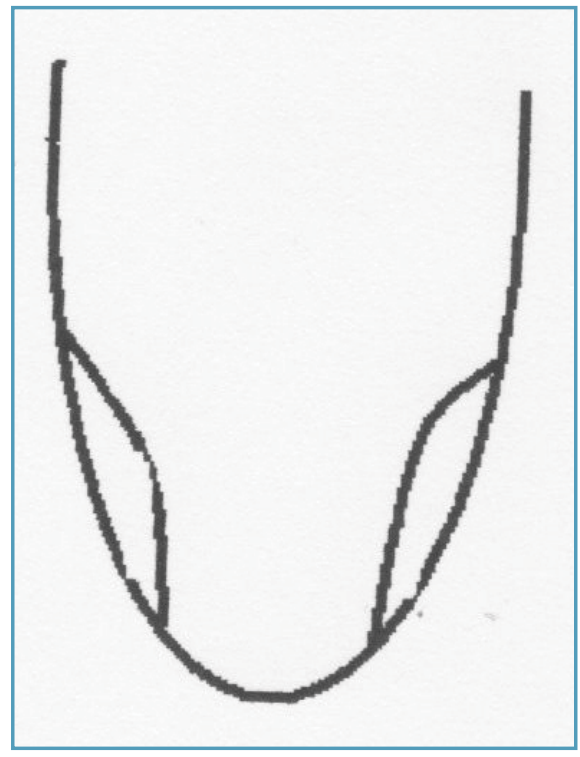

Abb. 2. Das vordere Drittel der Zunge wird der Brust zugeordnet. Eine Veränderung kann bei Frauen eine Erkrankung der Mammae widerspiegeln, nach [8].
Rücken- und Knieschmerzen, gespannter schneller Puls oder eine trockene, rote Zunge können Anzeichen für innere Hitze aufgrund von Yin- und Säfte-Mangel sein.

Bei einem Tumorgeschehen sind Blockaden durch Schleim, Blutstagnation und Bildung von Hitze-Toxinen involviert. Zum ohnehin schon gesteigerten Säftverbrauch und der eingeschränkten Produktion im Krankheitsverlauf können sich Flüssigkeitsverluste durch Operation, Erbrechen und Diarrhoe addieren. Die Diarrhoe tritt meist im Zuge von Radiatio - die Hitze und Feuer produziert - und/oder Chemotherapie auf. Der resultierende Substanzverlust in Kombination mit den geschädigten Yin-Säften kann zu einer Exazerbation des Karzinoms führen. Aus dieser Problematik entstand der Grundsatz in der Therapie: „Solange die Körpersäfte erhalten sind, ist die Prognose gut“ [1]. Das Behandlungsprinzip zielt dann darauf ab, das Yin zu nähren, Hitze zu klären, die Körpersäfte zu ergänzen und Trockenheit zu befeuchten.

\section{Diagnostik}

Primär ist es notwendig, den Tumor entsprechend schulmedizinischer Kriterien histopathologisch abzuklären und ein Bild über Grösse, Ausbreitung und Malignität zu erhalten.

Aus Sicht der TCM ist zu Behandlungsbeginn die Musterdifferenzierung essentiell. Laut der Chinesischen Medizin weisen Tumorpatienten einen (mehr oder weniger) palpablen Tumor auf, der als Masse Zheng Jia oder Ansammlung $J i J u$ bezeichnet wird. Da Ursache und Pathologie aber mannigfaltig sein können, ist es für die Praxis wichtig, das Grundprinzip zu definieren, welches die Krankheitsmatrix widerspiegelt und deren Verlauf bestimmt - unabhängig davon, wie vielfältig die Symptome sind.

Häufig stellt es eine gewisse Schwierigkeit dar, ein Grundmuster aus der komplexen Masse an klinischen Manifestationen zu isolieren. Besonders $\mathrm{Pa}$ tientinnen, die sich in einem fortgeschrittenen Krankheitsstadium befinden, bieten ein recht komplexes Bild. Einerseits Zeichen des lokalen Geschehens selbst, andererseits Allgemeinsymptome der progredienten Generalisierung, wie Erschöpfung, Kachexie, Dyspnoe, Appetitverlust, usw.

Eine Diagnose entsprechend TCMKriterien erstellt der Therapeut nach den vier Grundprinzipien: Befragung, Erkennen über Geruch und Gehör, Inspektion und Palpation [6]. Die chinesische Pulstastung ist für den Ungeübten schwierig, hingegen gehört zur Pal- pation auch das Abtasten der erkrankten Brust, denn dadurch können sich Hinweise auf die zugrunde liegende Pathologie ergeben [7].

- Hautfarbe: weisse Hautveränderung deutet auf Schleim hin, rote Hautveränderung deutet auf toxische Hitze hin.

- Mamillarsekret: gelb: Nässe-Hitze; eitrig: toxische Hitze; blutig/braun: Blut-Hitze und toxische Hitze.

- Tumorpalpation: weicher Knoten: Schleim; harter Knoten: Blut-Stase und/oder toxische Hitze; abgrenzbarkeit gut: Schleim; abgrenzbarkeit schlecht: toxische Hitze; verschiebbar: Schleim; nicht verschiebbar: Blut-Stase und/oder toxische Hitze.

\section{Zungendiagnostik}

Die Beobachtung der Zunge umfasst 4 Hauptkriterien: Farbe, Form des Zungenkörpers, Zungenbelag und -feuchtigkeit $[6,8]$.

- Die Farbe des Zungenkörpers zeigt den Zustand von Blut, Nähr-Qi, YinOrganen.

- Die Form weist auf den Zustand von Blut und Nähr-Qi hin.

- Der Belag gibt Auskunft über die Yang-Organe.

- Die Feuchtigkeit spiegelt den Zustand der Körperflüssigkeiten wider. 
Die topografische Aufteilung der Zunge erlaubt Aussagen über den Zustand der Funktionskreise. Die generelle Zuordnung der Organe auf der Zunge ist in Abb. 1 dargestellt.

Maciocia schreibt, dass eine Purpurfärbung im vorderen Drittel allgemein auf eine Blut-Stase im Thorax hinweist, wie bei Frauen mit Erkrankungen der Brust [8]. So ist bei einer Frau mit Brustkrebs die Purpurfärbung dieses Areals (das die Erkrankung der ipsilateralen Seite widerspiegelt) als prognostisch ungünstig anzusehen (Abb. 2). Die Prognose ist umso schlechter, je tiefer das Gebiet gefärbt ist. Generell gilt, dass sich die Zunge erst nach sehr langer Zeit livide verfärbt, so dass die Farbe in der Regel Zeichen für eine bereits lange bestehende Störung ist.

Damit besteht eine Kongruenz mit der Meinung von B. Kirschbaum, sie dokumentiert drei Fälle mit Mammakar- zinom [9]. Die drei Patientinnen weisen jeweils verschiedene Pathologien auf und damit auch unterschiedliche Zungen. In allen drei Fällen sind die beschriebenen Areale im vorderen Zungenbereich auffällig, d.h. gerötet. (Abb. 2)

All diese Zeichen sind Hinweis gebend, allerdings kann die seriöse endgültige Diagnose nur mit Hilfe westlicher Methoden (Mammographie, Histologie) gestellt werden.

\section{Literatur}

1. Maciocia G: Die Gynäkologie in der Praxis der chinesischen Medizin, Verlag für Ganzheitliche Medizin, Kötzting 2000

2. Flaws B: Screening for the potential for breast cancer according to traditional Chinese Medicine - A suggested research protocol. Vortrag. Boulder/USA.

http://www.datadiwan.de/netzwerk/index.htm ?/stiftung_paracelsus/sp_022d_.htm

3. Li Peiwen: Management of Cancer with Chinese
Medicine; Donica Publishing, St. Albans, Hertfordshire/UK 2003.

4. Schmit K: Onkologie in der TCM, Diplomarbeit im Rahmen des Lehrgangs für Traditionelle Chinesische Medizin an der Donau Universität Krems, 2005.

5. Krause B: Tumoren der Brust, Teil 3. Naturheilpraxis 2003;8:1102-1110

6. Maciocia G: Die Grundlagen der Chinesischen Medizin, Verlag für Ganzheitliche Medizin, Kötzting 1997.

7. Krause B: Tumoren der Brust, Teil 2. Naturheilpraxis 2003; 7:948-955.

8. Maciocia G: Zungendiagnostik in der chinesischen Medizin. ML Verlag, Uelzen 2000.

9. Kirschbaum B: Atlas und Lehrbuch der Chinesischen Zungendiagnostik, Band 1. Verlag für Ganzheitliche Medizin, Kötzting 1998.

\section{Anschrift der Autorin}

Dr. med. Kerstin Schmit, MSc

Master of Science in TCM

Aeskulap Klinik

Gersauerstrasse 8, CH-6440 Brunnen

kerstin.schmit@aeskulap.com

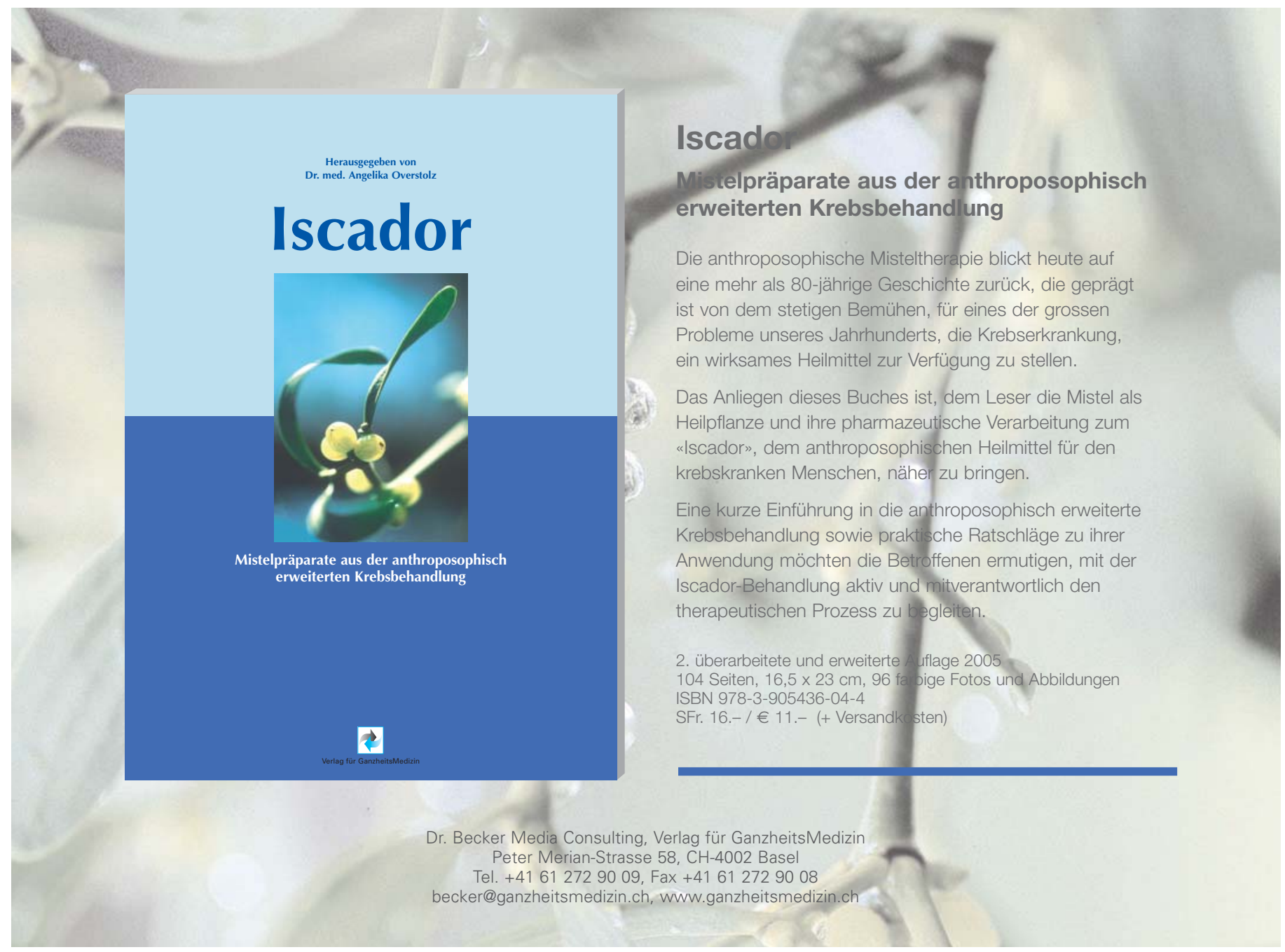

